\title{
Intakes of top shelf foods and contribution to energy and nutrients in a representative sample of school-aged children (5-12y) in Ireland
}

\author{
C. McGowan ${ }^{1,2}$, E. O’Sullivan ${ }^{1}$, L. Kehoe $^{2}$, A. Muldoon ${ }^{1}$, N. O’Kane ${ }^{5}$, J.V. Woodside ${ }^{5}$, \\ S. Watson ${ }^{5}$, A.P. Nugent ${ }^{5}$, M. Buffini ${ }^{3}$, J. Kearney ${ }^{4}$, B.A. McNulty ${ }^{3}$, A. Flynn ${ }^{2}$ and J. Walton ${ }^{1}$ \\ ${ }^{1}$ Department of Biological Sciences, Munster Technological University, Cork, Ireland, \\ ${ }^{2}$ School of Food and Nutritional Sciences, University College Cork, Cork, Ireland, \\ ${ }^{3}$ Institute of Food and Health, University College Dublin, Belfield, Dublin, Ireland, \\ ${ }^{4}$ School of Biological and Heath Sciences, Technological University Dublin, Dublin, Ireland and \\ ${ }^{5}$ Institute for Global Food Security, Faculty of Medicine, Health and Life Sciences, Queens University Belfast, Belfast, \\ $U K$
}

'Top-shelf foods' refer to foods/beverages high in calories, fat, sugar and salt, but low in fibre and micronutrients ${ }^{(1)}$. Healthy Eating Guidelines (HEG) in Ireland separate the top shelf from lower shelves to indicate that these foods/beverages are not needed for good health and consumption should be limited to 1-2 times/week ${ }^{(2)}$. The aim of this study was to investigate the intake of 'top-shelf foods' and their contribution to energy and nutrient intakes in school-aged children in Ireland.

Analyses were based on data from the National Children's Food Survey II (NCFS II) (2017-18) (www.iuna.net). A 4-day weighed food record was used to collect food/beverage intake data from 600 children (5-12y). Nutrient intakes were analysed using UK and Irish food-composition data. 'Top-shelf foods' were defined according to the top-shelf of the HEG in Ireland and processed foods (e.g. takeaways/ready-meals) were excluded as these foods are often from a combination of the shelves ${ }^{(2)}$. The contribution of 'top-shelf foods' to overall intakes of energy and nutrients was calculated using SPSSC V26.

'Top-shelf foods' were consumed by all children at least once over the 4 days. Eighty-one percent of children consumed 'top-shelf foods' on all 4 days, $14 \%$ on 3 days only, $4 \%$ on 2 days only and $1 \%$ on just one day. Overall, $80 \%$ of children consumed 'biscuits, including crackers', 67\% consumed 'soft-drinks' (with/without added sugar), 64\% consumed 'crisps, popcorn \& other savoury-snacks', $64 \%$ consumed 'sugars, sweeteners, preserves \& chocolate spreads', 59\% consumed 'chocolate confectionery', 57\% consumed 'cakes, pastries \& buns', 57\% consumed 'creams, ice-creams \& desserts', 40\% consumed non-chocolate confectionery (e.g. sweets/jellies) and $18 \%$ consumed 'cereal-bars'. 'Top-shelf foods' contributed on average $21 \%$ of energy intake, comprised of: 'biscuits, including crackers' (5\%), 'cakes \& pastries' (4\%), 'creams, ice-creams \& desserts' (3\%), 'crisps, popcorn \& savoury-snacks' (3\%), 'chocolate confectionary' ( $3 \%$ ) and 1\% each from 'soft-drinks' (with/without added sugar), 'non-chocolate confectionery', 'sugars, sweeteners, preserves $\&$ chocolate spreads' and 'cereal-bars'. 'Top-shelf foods' contributed to intakes of protein ( $8 \%)$, total fat (24\%), carbohydrate (24\%) and dietary fibre (13\%). 'Top-shelf foods' also contributed to intakes of saturated fat (26\%), MUFA (24\%), PUFA (20\%), free sugar $(61 \%)$ and salt $(14 \%)$. 'Top-shelf foods' contributed greater proportions of nutrients with increasing frequency of consumption (no of days), however these findings should be interpreted with caution due to small numbers in some groups.

This study found that 'top-shelf foods' were consumed by all children and more often than recommended. 'Top-shelf foods' made significant contributions to intake of energy (21\%) (similar contributions from each category) and also contributed to significant proportions of total fat $(24 \%)$, saturated fat $(26 \%)$, free sugar $(61 \%)$ and salt $(14 \%)$. These preliminary results will be useful for informing strategies to reduce the intake of top-shelf foods in school-aged children in Ireland.

\section{Acknowledgements}

This study was funded by: safefood The Food Safety Promotion Board. The National Children's Food Survey II was funded by the Department of Agriculture, Food and the Marine in Ireland.

\section{References}

1. Food Safety Authority of Ireland (FSAI) (2016) A guide supporting the Food Pyramid.

2. Department of Health (DOH) (2016) Healthy Food for Life - the Healthy Eating Guidelines and Food Pyramid. 